\title{
Due pittrici greche nel De mulieribus claris (I): Tamari'
}

\author{
Francisco José Rodríguez Mesa \\ Universidad de Córdoba \\ francisco.rodriguez.mesa@uco.es \\ https://dx.doi.org.10.12795/futhark.2018.il3.06
}

Fecha de recepción: 3.12 .2018

Fecha de aceptación: 20.12 .2018

Riassunto: Fra le centosei biografie muliebri che compongono il De mulieribus claris di Giovanni Boccaccio si annovera un certo gruppo di donne che spiccarono per la loro saggezza o per il loro virtuosismo in diverse arti. All'interno di questa categoria, il certaldese narra brevemente il talento di due pittrici che vissero nella Grecia dell'Antichità: Tamari, figlia di Micone (capitolo LVI), e Irene, figlia di Cratino (LIX). II presente articolo - primo di due studi che prenderanno in analisi le due vite suddette - studia le particolarità del capitolo dedicato a Tamari (LVI) analizzando le fonti utilizzate dall'autore e la struttura della vita, che viene paragonata agli altri modelli presenti nella silloge, sia per quanto riguarda l'estensione, sia rispetto ai motivi per i quali eccelse la protagonista.

Parole chiavi: Boccaccio; De mulieribus claris; donne nel Medioevo; letteratura esemplare femminile; artiste nel Medioevo.

\section{Two Greek Paintresses in the De Mulieribus Claris (I): Timarete}

\begin{abstract}
Among the one hundred and six lives of exemplary women conforming Giovanni Boccaccio's De mulieribus claris, there is a group devoted to female characters who excelled in virtue of their wisdom or their talent in different arts. Inside this category, the author narrates briefly the lives of two Ancient Greek paintresses: Timarete, Micon's daughter (chapter LVI) and Eirene, Cratinus's daughter. In this article - which is the first one of a series of two studies about the above-mentioned lives - I analyse the particular features of the chapter devoted to
\end{abstract}

1 Quest'articolo si inserisce nel Progetto di Ricerca del Ministerio de Economía y Competitividad Spagnolo "Voces de mujeres en la Edad Media: realidad y ficción (siglos XII-XIV)", FFI20I4-55628-P. 
Timarete (LVI) focusing on its sources and its structure and comparing the latter to other schemes used by Boccaccio in the same work. This comparison takes into account both the length of the biographies and the virtues that made the paintress stand out.

Keywords: Boccaccio; De mulieribus Claris; Medieval women; Exemplary women tradition; Women artist in the Middle Ages.

Sumario: Entre las ciento seis biografías femeninas que conforman el De mulieribus claris de Giovanni Boccaccio se encuentra un cierto grupo de mujeres que destacaron por su sabiduría o por su virtuosismo en distintas artes. En el seno de esta categoría, el autor narra brevemente el talento de dos pintoras de la Grecia Antigua: Tamaris, hija de Micón (capítulo LVI), e Irene, hija de Crátino. El presente artículo -primero de dos estudios que analizarán las vidas boccaccescas de las citadas pintoras- estudia las particularidades del capítulo dedicado a Tamaris (LVI), analizando sus fuentes y la estructura de la biografía y comparándolas con otros modelos presentes en el catálogo de vidas, tanto por lo que respecta a la extensión como por lo que concierne a los motivos por los que destacó la protagonista.

Palabras clave: Boccaccio; De mulieribus claris; mujer en la Edad Media; literatura ejemplar; mujeres artistas en la Edad Media.

Come dimostrò Ricci (1959), verso il |36| Giovanni Boccaccio comincia a lavorare alla stesura di una silloge di vite di donne famose che porterà come titolo De mulieribus claris. La molteplicità di manoscritti che tutt'oggi si conserva e la fortuna dei cataloghi di biografie femminili dei secoli successivi provano che quest'opera procurò un enorme successo al certaldese, che con la raccolta si pose come scopo quello di rimediare alla mancanza di antologie di vite muliebri all'interno di un genere che ebbe nel De viris illustribus di Petrarca il proprio capostipite.

Sebbene la fortuna di cui godette il DMC dal Quattrocento in poi fosse tutt'altro che discreta, il numero di studi critici che sono stati dedicati a quest'opera di Boccaccio si può continuare a dire esiguo se paragonato con la minuziosità con cui altre opere del certaldese sono state analizzate. In effetti, da quando Hortis (1877) cercò di attirare l'attenzione degli studiosi su questo lavoro e qualche decennio dopo Laura Torretta insistette sul valore della silloge (I902a e 1902b), non sono state molto numerose le pubblicazioni che hanno analizzato questa antologia in maniera non collaterale. Vale a dire, continuano ad essere scarsi i lavori in cui il DMC venga analizzato da sé e per sé e non come serbatoio di 
citazioni più $\circ$ meno superflue e più $\circ$ meno utili nel ragionare su altri lavori boccacceschi.

Tra gli studi che analizzano il $D M C$ con un approccio più generale $\circ$ più ampio spiccano quelli Cerbo (1974), Jordan (1987), Müller (1992a), Kolsky (1993; 2003) e Filosa (20I2). Si occupano di questioni intertestuali Müller (1992b), Sciacovelli (2003) e Filosa (2004; 2006). Analizzano i manoscritti contenenti l'opera Zaccaria (1963), Zappacosta (1973), Branca-Zaccaria (1996) e Nuvoloni (2003). Aurigemma (1988-1990) e Filosa (2007) studiano l'opera da un punto di vista storico, ancora Zaccaria (1965) e Auzzas (2002-2003) impostano le proprie ricerche sulla base della lingua adoperata da Boccaccio e sempre Zaccaria (1978), Meale (1992), Kolsky (2005) e Caputo (2008) analizzano la fortuna della silloge.

Nel paragrafo precedente si trova una parte importante delle opere critiche consacrate in modo esclusivo al DMC dalla metà del Novecento ai giorni nostri. Se pensiamo che si tratta di una silloge composta da uno degli scrittori italiani più influenti nella letteratura occidentale di tutti $\mathrm{i}$ tempi, si potrebbe giungere alla conclusione che l'interesse che quest'antologia ha suscitato tra i critici odierni è ben lontano dalla fruizione con cui essa fu letta e imitata durante il periodo in cui si diffuse, poco dopo la sua stesura.

Un'ulteriore prova dell'isolamento al quale la raccolta è stata condannata nella storia recente può trovarsi nel numero di traduzioni in diverse lingue: la prima traduzione moderna in inglese dell'opera, fatta da Guarini, risale al $1963^{2}$, la traduzione italiana, di Zaccaria, è del 1967 e la versione spagnola, di Díaz-Corralejo, non vide la luce fino al 2010.

Nonostante il limitato numero di studi incentrati sul $D M C$, le vite dei personaggi più noti sono già state studiate con una certa sistematicità, con l'indicazione di interpretazioni, fonti precedenti, modifiche boccaccesche e l'eventuale successo nelle opere che sorgono nella scia della silloge. Tuttavia, fra le centosei biografie muliebri che il certaldese annovera nell'ultima versione della raccolta, ne spiccano due, poste assai vicine l'una all'altra, che hanno dei tratti in comune e che finora non sono mai state analizzate dalla critica in modo individuale. Si tratta di quelle di Tamari e di Irene.

Sia Tamari, protagonista della biografia LVI, sia Irene, la cui vita si narra nel capitolo LIX, furono due importanti pittrici della Grecia classica, figlie di due reputati pittori dai quali appresero quella professione che riuscirono a dominare con una padronanza tale da superare i loro padri e maestri. Questo fatto, narrato nel DMC, proviene dal libro XXXV della Storia naturale di Plinio, testo che il

${ }^{2}$ Dal 1963 altre due traduzioni inglesi sono state pubblicate, sempre negli Stati Uniti, la prima di Virginia Brown (2003) e la seconda di Guido Guarino (201I). 
certaldese usa come fonte principale per i capitoli contenenti le vite di entrambe le pittrici. Tuttavia, oltre alle origini geografiche e alle circostanze delle loro famiglie, le biografie di Tamari e di Irene mostrano un'altra caratteristica comune che acquisisce una certa rilevanza se opposta alla prassi abituale della silloge: si tratta di capitoli di una straordinaria - e anomala - brevità.

Tenuto conto di questi fattori, con il presente articolo - che non è che la prima parte di un insieme di due studi nei quali si analizzeranno le vite delle due sole pittrici presenti nel DMC - ci proponiamo di determinare le diverse parti in cui la biografia di Tamari può essere suddivisa per mettere in rapporto questa microstruttura con quella predominante nella silloge. Nello stesso modo, si studieranno l'accuratezza e la fedeltà con cui Boccaccio adopera la fonte pliniana nella propria versione della biografia di Tamari e si presterà un'attenzione particolare agli elementi provenienti dall'invenzione del certaldese per cercar di spiegare i possibili motivi che portarono l'autore a manipolare la fonte latina in questo modo.

Nel DMC può essere individuata una serie di biografie muliebri che hanno in comune il fatto che le loro protagoniste sono delle intellettuali o che, in qualche modo, svolgono dei lavori di creazione o di studio in cui hanno tradizionalmente spiccato gli uomini. Per quanto riguarda le artiste che Boccaccio annovera nella silloge, oltre a Tamari ed Irene, bisogna citare le poetesse Saffo (LVII) e Cornificia (LXXXVII), le letterate Leonzio (LX) e Proba (XCVII) e la scultrice e pittrice Marzia (LXVI). Come ha già indicato Filosa (2012: 169),

L’innovazione più evidente [...] delle eroine del De mulieribus è senz'altro la celebrazione di pittrici, scultrici, scrittrici e, in generale, di figure intellettuali. La semplice presenza di queste donne nella compagine testuale è una novità assoluta rispetto alle catalogazioni del passato (con la parziale eccezione della lettera [Familiare XXI 8] di Petrarca che [...] potrebbe aver influenzato la stesura dell'opera di Boccaccio). È fuor di dubbio che tale inclusione sia da ascrivere agli interessi umanistici di messer Giovanni: la donna del nuovo mondo comporrà poesie (come Saffo e Cornificia), saprà latino e greco (come Proba), sarà un'intellettuale (come Carmenta e Nicaula); dipingerà (come Tamiri $\left[\mathrm{sic}^{3}\right]$ ed Irene), scolpirà (come Marcia [sic]), inventerà qualcosa di utile per il genere umano (come Minerva, Cerere, Iside e Panfila), avrà a cuore l'amicizia e l'etica civile.

Le vite a cui la studiosa fa riferimento in questo paragrafo condividono un'importante caratteristica se contrapposte alle tendenze che si mostrano predominanti nella silloge. Nella raccolta si trova una massiccia presenza di dee o

${ }^{3}$ Data la descrizione della professione del personaggio, interpretiamo che Filosa intende parlare della pittrice Tamari anziché di Tamiri, regina degli sciti e protagonista della biografia XLIX. 
regine $^{4}$ che, non bisogna dimenticarlo, diventano esemplari per il modo in cui esercitano quel potere che è a loro connaturale e che, di solito, hanno ereditato. In opposizione a questo, le artiste e le intellettuali spiccano e sono degne di eterno ricordo per aver saputo svolgere in maniera egregia un'attività che si basa esclusivamente sulle proprie abilità. In altre parole, mentre le regine, per il semplice fatto di esserlo, hanno già a disposizione tutti gli elementi che, se adoperati nel modo giusto, portano alla fama e all'esemplarità, le artiste sono artefici del proprio successo poiché sono esse stesse a sviluppare questi strumenti e a utilizzarli in modo tale da essere ricordate dai posteri.

D'altronde e dal punto di vista di un'eventuale indipendenza della donna, c'è un altro fattore che bisogna aver presente per quanto riguarda i due gruppi di vite muliebri a cui si è appena fatta allusione, ed è quello del merito o della "responsabilità" che l'uomo potrebbe avere nell'esemplarità femminile. Non c'è bisogno di insistere sul fatto che nel DMC la donna è solita a presentarsi secondo il proprio rapporto con l'uomo. In effetti, i paratesti che precedono le biografie indicano molto frequentemente rapporti di parentela fra le protagoniste e uomini celebri. Tuttavia, i capitoli della silloge potrebbero anche essere divisi in due gruppi a seconda dell'influenza che questi uomini hanno potuto esercitare sulle protagoniste e sulla loro fama.

Da un lato, e nel caso delle sovrane, si tratta di personaggi che, come è già stato detto, hanno ricevuto il loro potere - cioè, lo strumento che le ha fatte diventare famose - dagli uomini che le hanno precedute sul trono. In questo caso lo strumento è ereditato, vale a dire, trasmesso al di là delle caratteristiche intrinseche e della volontà dell'erede, che diventa un mero destinatario passivo di questo elemento.

Dall'altra parte, e per quanto riguarda le artiste e le intellettuali, è vero che molto spesso appartengono ad un lignaggio di professionisti eccellenti in un determinato ambito; basti pensare ai paratesti che presentano e precedono le vite delle due pittrici: "De Thamari Myconis filia" (LVI) e "De Yrene Cratini filia" (LIX). Ciononostante, è palese che il talento e l'abilità genitoriale non si trasmette alle figlie con la stessa immediatezza, passività e mancanza di volontà dei titoli regali. In effetti, se si diceva che le regine non erano altro che passivi ricettori dei meriti degli antenati, nel caso delle abilità e le perizie artistiche il merito conquistato dall'antenato dev'essere rinnovato e riacquistato in ogni generazione, in modo tale che la donna che spicca nell'arte o nello studio è ben lungi dall'essere una figura

${ }^{4}$ Si ricordi che, come conseguenza dell'evemerismo (che è un ingrediente fondamentale nelle opere umaniste di Boccaccio e in modo particolare nel DMC e nella Genealogia), molto spesso le divinità grecoromane sono identificate con eroi, sovrani o altri personaggi storici le cui gesta furono importanti per lo sviluppo delle civiltà a cui appartenevano. 
passiva o la semplice ricettrice di un'eredità: essa deve lavorare e battersi per fare suo il talento delle generazioni della propria stirpe che l'hanno preceduta, ed è proprio tramite questo sforzo che porta alla (ri)conquista e alla padronanza delle abilità artistiche o intellettuali che diventa degna di encomio e ricordo nell'avvenire.

La differenza che è stata appena indicata fra entrambi i tipi di esemplarità comporta un ragionamento che può dirsi tutt'altro che secondario: dal momento in cui il proprio sforzo - e non l'eredità - può portare alla fama e all'esemplarità, queste sono alla portata delle donne - e degli uomini - comuni al di là della loro classe sociale e al di là del loro rapporto con il ceto dirigente. Questa è, a nostro avviso, la principale novità e uno dei principali segni di modernità del DMC: dal momento in cui Boccaccio decide di includere biografie di donne che hanno raggiunto la fama per merito proprio accanto a vite di sovrane e nobildonne, contribuisce alla democratizzazione del genere e, con essa, all'esaltazione del libero arbitrio 5 .

Una volta contestualizzata l'opera e le implicazioni delle biografie delle pittrici, passiamo ad analizzare il racconto della vita di Tamari. Uno dei principali aspetti che ci colpisce di questo capitolo LVI è la sua brevità. In effetti, in contrasto con le lunghe esposizioni e dissertazioni su altre donne, Boccaccio si limita a raccontare di Tamari, figlia di Micone, quanto segue (Boccaccio 1967: 226-228):

Thamaris mulier evo suo pictrix egregia fuit; cuius virtus, etsi forsan veternositas plurimum abstulerit, nomen tamen egregium nec artificium adhuc abstulisse potuit. Volunt igitur hanc nonagesima olympiade filiam fuisse Myconis pictoris; verum cuius, cum duos fuisse Mycones et ambo pictores et eodem tempore Athenis floruisse legamus, non distinguunt, nisi his paucis verbis eam filiam fuisse Myconis cui minoris cognomen additum ferunt. Sane cuiuscunque fuerit, tam miro ingenio, despectis muliebribus officiis, paternam artem imitata est ut, regnante apud Macedonas Archelao, singularem picture gloriam adepta sit, in tantum ut Ephesi, apud quos honore precipuo Dyana colebatur, eiusdem Dyane effigiem, in tabula quadam mano eius pictam, tanquam celebrem servaverint diu. Que cum in longissimam etatem perseverasset, artificii huius testimonium tam grande prebuit, ut in hodiernum usque memorabile videatur: equidem laudabile plurimum, si prospectemus fusos et calathos aliarum.

La narrazione della vita della pittrice può essere suddivisa nelle seguenti sette parti:

I. Presentazione: "Thamaris mulier evo suo pictrix egregia fuit".

${ }^{5}$ Per approfondire la presenza delle artiste e intellettuali nella silloge si veda Müller (1992a: 128-145) e Filosa (2012: 168-172). 
2. Motivi per i quali va ricordata: "cuius virtus, etsi forsan veternositas plurimum abstulerit, nomen tamen egregium nec artificium adhuc abstulisse potuit".

3. Epoca: "Volunt igitur hanc nonagesima olympiade[...]"

4. Genealogia: "[...] filiam fuisse Myconis pictoris; verum cuius, cum duos fuisse Mycones et ambo pictores et eodem tempore Athenis floruisse legamus, non distinguunt, nisi his paucis verbis eam filiam fuisse Myconis cui minoris cognomen additum ferunt".

5. Professione ed epoca di svolgimento: "Sane cuiuscunque fuerit, tam miro ingenio, despectis muliebribus officiis, paternam artem imitata est ut, regnante apud Macedonas Archelao, singularem picture gloriam adepta sit $[\ldots]$ ".

6. Capolavoro: "in tantum ut Ephesi, apud quos honore precipuo Dyana colebatur, eiusdem Dyane effigiem, in tabula quadam mano eius pictam, tanquam celebrem servaverint diu. Que cum in longissimam etatem perseverasset, artificii huius testimonium tam grande prebuit, ut in hodiernum usque memorabile videatur".

7. Paragone con le altre donne: “[...] equidem laudabile plurimum, si prospectemus fusos et calathos aliarum".

Ricarda Müller dedica un capitolo del suo libro sul DMC (1992a: 36-46) alla costruzione delle vite nell'opera e, rispetto agli incipit dei capitoli, afferma (1992a: 37):

Zu Beginn eines jeden Kapitels gibt Boccaccio Grundinformationen über die Frauengestalt, die er beschreiben will. Dazu gehören die nationale Herkunft, die Familie - d.h. der Vater oder der Ehemann - und die Zeit, in der lebte.

Diese Angaben sind nicht immer vollständig - je nachdem, welche Informationen Boccaccio vorliegen hatte.

Tutti questi elementi possono essere rintracciati nella vita di Tamari, tanto che pochi altri possono essere rinvenuti nella biografia della pittrice. Certamente, tra le sette sequenze suddette, ben quattro (quelle numerate I, 3, 4 e 5) si annoverano fra i punti che Müller individua come tipici della presentazione di ogni capitolo (lasciando a un lato il vero e proprio svolgimento delle vite). Questo indica che, data la brevità della biografia, quasi la metà del testo ha come scopo testimoniare chi fosse questa donna degna di ricordo, quando e dove visse e chi fosse suo padre. Oltre a questo, pochi sono i dati che Boccaccio fornisce e questi non vanno al di là della professione che svolse, quella che l'ha fatta diventare una mulier clara.

Come afferma Franklin, "Tamaris (LVI) is remembered for painting an image of the virginal goddess Diana" (2006: 48). II fatto che il capolavoro che si cita della 
pittrice sia proprio un dipinto della dea Diana non ci sembra casuale. È vero che come si vedrà più avanti - Boccaccio non è il primo autore a menzionare questo quadro; anzi, già Plinio, che è la fonte principale del certaldese per questa biografia, ne parla.

Diana, omologa nella mitologia romana dell'Artemide greca, era la protettrice delle selve, degli animali selvaggi, delle fonti e dei torrenti, ma anche delle donne fino al punto di essere considerata l'emblema della castità femminile. Vale a dire, di una qualità che Boccaccio pone al centro delle virtù attribuibili alle donne più eccelse del $D M C$. In effetti, numerose sono le donne della silloge che spiccano per la loro castità, per la loro verginità o per la loro rettitudine morale, si pensi a Orizia e Antiope, regine delle amazzoni $(X I X-X X)$, alla sibilla Eritrea o Erifile $(X X I)$, alla sibilla Almatea o Deifele $(X X V I)$, a Manto $(X X X)$, a Camilla (XXXIX), a Didone (XLII), a Lucrezia (XLVIII), a Tamiri (XLIX), a Ippona (LIII), ad Artemisia (LVII), a Virginia, figlia di Virginio (LVIII), a Virginia, moglie di Lucio Volunnio (LXIII), a Marzia (LXVI), a Sulpicia (LXVII), alla moglie di Orgiagonte (LXXIII), a Gualdrada (CIII) e a Costanza (CIV). Nonostante questo vasto elenco, nel $D M C$ si può osservare anche la tendenza opposta, cioè, fra quelle donne che emergono come esempi negativi, la lussuria è il vizio più comune. Ne sono esempi palesi le vite di Semiramide (II), Venere (VII), Rea llia (XLV), Olimpiade (LXI), Flora (LXIV), Cleopatra (LXXXVIII), Faustina Augusta (XCVIII) o Semiamira $(X C I X)^{6}$.

Riflettendo sull'importanza della castità a livello globale nel $D M C$ e mettendolo in rapporto con gli esigui elementi che Boccaccio offre per la caratterizzazione di Tamari, spicca il fatto che il certaldese abbia scelto come una delle protagoniste della sua antologia una donna il cui capolavoro è proprio un'effigie della dea della castità. Questo fatto, a nostro avviso, colloca Tamari in una posizione superiore alle altre donne esemplari perché caste: la pittrice non solo è in grado di eccellere per la propria abilità, ma tramite questo talento riesce a ricreare un oggetto che diventerà esso stesso emblema della principale virtù muliebre e, come tale, venerato per secoli fra un popolo - gli efesini - che già si ricorda per la devozione a Diana. In altre parole, mentre grazie al proprio atteggiamento casto e virginale una parte importante delle donne del DMC sembra imitare Diana, Tamari non la imita, ma la (ri)crea, diventando così una sorta di "artifex claritatis".

\footnotetext{
${ }^{6}$ L'importanza della castità femminile come strumento di esemplarità si sviluppa nella maggior parte dei casi intorno a due ambiti: la donna sposata o la donna vedova. Ė vero che nella silloge ci sono certe protagoniste - a partire da Venere stessa - che hanno a che vedere con l'esercizio della prostituzione, ma le vite più ricche di sfumature per quanto riguarda la moralità muliebre sono quelle che hanno come protagoniste donne maritate o i cui coniugi sono morti. Per approfondire in alcuni di questi capitoli, si veda Müller 1992a: II2-127; 154-160.
} 
Si è già parlato del rapporto che collega la biografia boccaccesca di Tamari a Plinio e alla sua Storia naturale. Effettivamente, è noto che il libro XXXV dell'opera pliniana è alla base della vita della pittrice, fino al punto che quasi tutte le informazioni che fornisce il $D M C$ vi possono essere rintracciate ${ }^{7}$. All'interno del suddetto libro due sono i capitoli che Boccaccio sembra adoperare per la costruzione della sua biografia esemplare.

II secondo dei brani utilizzati si trova nella conclusione del capitolo $\mathrm{XL}$, esattamente si tratta del frammento 147, dove Plinio elenca le donne pittrici:

Pinxere et mulieres: Timarete, Miconis filia, Dianam, quae in tabula Ephesi est antiquissimae picturae; Irene, Cratini pictoris filia et discipula, puellam, quae est Eleusine, Calypso, senem et praestigiatorem Theodorum, Alcisthenen saltatorem; Aristarete, Nearchi filia et discipula, Aesculapium. laia Cyzicena, perpetua uirgo, M. Varronis iuuenta Romae et penicillo pinxit et cestro in ebore imagines mulierum maxime et Neapoli anum in grandi tabula, suam quoque imaginem ad speculum. (Plinio 1985: 99)

Come si può vedere, se si paragona questo brano alla vita boccaccesca di Tamari, la prima frase pliniana sembra essere stata la principale testimonianza che il certaldese adoperò per la stesura di quelle parti che prima sono state numerate come $4^{8}$ e, soprattutto, 6 , dove si individua il capolavoro della pittrice.

Più particolare e meno rispettoso della lettura della fonte pliniana è l'utilizzo che Boccaccio fa del primo brano della Storia naturale, che prende come base per la biografia. Il paragrafo XXXV del libro XXXV di Plinio discorre degli artisti operanti prima della novantesima Olimpiade e, parlando di Polignoto di Taso, si afferma

Huius est tabula in porticu Pompei, quae ante curiam eius fuerat, in qua dubitatur ascendentem cum clupeo pinxerit an descendentem. Hic Delphis aedem pinxit, hic et Athenis porticum, quae Poecile uocatur, gratuito, cum partem eius Micon mercede pingeret. Vel maior huic auctoritas, siquidem Amphictyones, quod est publicum Graeciae consilium, hospitia ei gratuita decreuere. Fuit et alius Micon, qui minoris cognomine distinguitur, cuius filia Timarete et ipsa pinxit. (Plinio 1985: 62-63)

Come si apprende da questo brano, Micone il minore, padre di Timarete secondo Plinio (e probabile padre dell'artista secondo Boccaccio), visse e lavorò prima della novantesima Olimpiade. Tuttavia, Boccaccio posticipa la nascita di Tamari fino alla novantesima Olimpiade stessa: "Volunt igitur hanc nonagesima olympiade filiam fuisse Myconis pictoris".

7 Tutte le traduzioni moderne dell'opera indicano tramite il proprio apparato paratestuale questa filiazione.

${ }^{8}$ Almeno l'incipit di 4, cioè, l'identificazione del nome del padre di Tamari. 
II brano pliniano qui riprodotto corrisponde alla conclusione del $X X X V$ capitolo del XXXV libro dove, com'è stato detto, si espone l'arte prima della novantesima Olimpiade. Subito dopo, però, Plinio elenca gli artisti operanti durante questa Olimpiade: "LXXXX autem olympiade fuere Aglaophon, Cephisodorus, Erillus, Euenor, pater Parrhasii et praeceptor maximi pictoris, de quo suis annis dicemus" (Plinio 1985: 63). Se si considera che l'allusione alla novantesima Olimpiade compare subito dopo il nome di Tamari, è alquanto probabile che Boccaccio leggesse erroneamente questo brano (come già notò Torretta, 1902a: 285-286). Un'altra possibilità che non si può scartare è che il manoscritto che l'autore adoperò fosse corrotto o non mostrasse la separazione fra quelli che oggi consideriamo capitoli XXXV e XXXVI del libro pliniano.

Al di là di questo malinteso rispetto alla Storia naturale, di questo passaggio Boccaccio ricava le informazioni per la stesura dei brani 3, 4 e 5 della vita di Tamari; cioè, le parti in cui il certaldese individua il padre (per opposizione all'altro Micone) e l'epoca della pittrice. Quest'analisi della fonte implica che, oltre ai brani I e 2 che si limitano ad individuare la professione e il talento notevole della protagonista, c'è un'altra - ed essenziale - aggiunta dell'autore: il brano 7.

Quest'ultima parte della biografia, che si limita ad una sola frase ("equidem laudabile plurimum, si prospectemus fusos et calathos aliarum"), si colloca come corollario alla vita di Tamari e ha come scopo valorizzare la sua esistenza e la persistenza nel ricordo dei lavori che riuscì a portare a termine opponendoli alle mansioni di cui generalmente si occupano la maggior parte delle donne. II certaldese afferma che l'effigie di Diana fatta dalla pittrice costituisce una testimonianza molto lodevole se consideriamo le ceste e i fusi per filare delle altre donne ${ }^{9}$. A nostro avviso, quest'affermazione non andrebbe interpretata come una critica ai lavori manuali di cui le donne comuni si occupavano ai tempi dell'autore, bensì come un giudizio che riguarda il valore delle arti in quanto risultato di questi lavori manuali: la pittura è una delle arti più importanti e, dunque, il pittore eccelle sugli altri artigiani.

È già stata sottolineata la disparità nell'estensione dei diversi capitoli che conformano il DMC. In questo senso, Müller non soltanto stabilì una divisione che

\footnotetext{
${ }^{9}$ La traduzione di questo brano ha causato alcune difficoltà nelle versioni moderne del DMC fino a punto che certi traduttori hanno cambiato drasticamente il significato di questa frase e, quindi (ed ecco il vero problema), l'interpretazione globale di questa biografia. Così, Díaz-Corralejo, nella versione spagnola, traduce "tan digno de ser muy alabado como si miramos los husos o las canastillas de labor de las demás mujeres" (Boccaccio 20I0: 228. II corsivo è nostro) ["così degno di essere lodato come se guardiamo i fusi o le ceste per filare delle altre donne"]. Questa costruzione che implica una comparazione di uguaglianza è assai lontana dall'interpretazione che si deriva dal testo originale del Boccaccio, che ha come scopo far eccellere la figura di Tamari, tramite la propria opera, sul resto delle donne.
} 
aveva a che vedere con la lunghezza di ogni biografia, ma anche per quanto riguarda la qualità o l'elaborazione delle vite. Per dirlo con le sue parole, "es gibt sehr lange und sehr kurze Kapitel, relativ schlicht zusammengestellte Materialsammlungen und sorgfältig ausgefeilte Stücke” (1992a: 36).

Nel caso della vita di Tamari non bisogna dimenticare che si tratta, effettivamente, di un capitolo che si può annoverare fra i più brevi della silloge, ma è anche doveroso ricordare che questa brevitas all'interno dell'ambito del DMC comporta, allo stesso tempo, il risultato di una notevole procedura di amplificatio rispetto alla fonte pliniana, dove - come si è visto - le allusioni alla vita di Tamari si riducono a poche parole.

Se, tenendo presente quest'idea, si ritorna alla duplice divisione quantitativa e qualitativa abbozzata da Müller e si riflette sul fatto che, ai tempi di Boccaccio, Tamari era ben lontana dall'essere un personaggio conosciuto ai livelli di quelle donne celebri che occupano le biografie più ampie ${ }^{10}$ si potrebbe pensare che il certaldese attuasse mosso da una doppia motivazione nel momento in cui scelse le vite da includere nella sua silloge. Da una parte, l'autore avrebbe scelto donne ampiamente conosciute, per le cui vite avrebbe consultato e combinato molteplici fonti e le cui biografie avrebbero contribuito a rendere l'opera più narrativa e piacevole. Dall'altra parte, avrebbe anche scelto un numero di donne molto meno note le cui vite sarebbero descritte in un modo assai più sintetico, basandosi su una quantità minore di fonti e che avrebbero dotato l'antologia di una componente più enciclopedica e teorica.

In altre parole, fra le donne riunite nel $D M C$, quelle che sono già famose vengono romanzate, mentre quelle che non lo sono o che lo sono in un grado minore vengono soltanto definite. Non bisogna dimenticare che entrambe le pittrici (Tamari ed Irene) appartengono a questa seconda categoria come succede anche con il resto delle donne artiste o intellettuali. Questo è, dal nostro punto di vista, uno dei principali valori della silloge, perché con queste inclusioni a modo di pillole teoriche nascoste tra i piaceri della lettura delle vite di donne più celebri, Boccaccio contribuisce a ricuperare e a far conoscere fra un pubblico femminile figure veramente esistite nel passato, che spiccano per il proprio talento e che, senza aver ereditato alcun tipo di potere politico, si presentano come artefici del proprio destino.

In conclusione, la sola presenza della vita di Tamari nel DMC emerge come una prova definitiva della modernità dello scopo della raccolta boccaccesca, soprattutto perché in questa biografia si viene a narrare la vita di una donna che

${ }^{10}$ Si pensi, ad esempio, a Didone (XLII), Artemisia (LVII), o Cleopatra (LXXXVIII), protagoniste delle biografie più lunghe.

Futhark I3 (2018) 
divenne esemplare grazie al proprio lavoro e che riuscì perfino a eclissare la figura maschile che in esso l'aveva preceduta: quella di suo padre, la cui identità Boccaccio nemmeno conosce con esattezza.

\section{Bibliografia}

Aurigemma, M. (1988-1990). Boccaccio e la storia. Osservazioni sul De claris mulieribus. Humanitas e poesia: 85- 102.

Auzzas, G. (2002-2003). Appunti sull'onomastica 'greca' del Boccaccio. Studi Petrarcheschi I2-I3: 24I-256.

Boccaccio, G. (1963). Concerning Famous Women, trad. Guido Guarino. New Brunswick: Rutgers University Press. (1967). De mulieribus claris, ed. Vittorio Zaccaria. Milano: Mondadori. - (2003). Famous Women, trad. Virginia Brown. Cambridge: Harvard University Press.

(20I0). Mujeres preclaras, trad. Violeta Díaz-Corralejo. Madrid: Cátedra. . (20II). On Famous Women, trad. Guido Guarino. New York: Italica Press.

Branca, V. e Z., V. (1996). Un altro codice del De mulieribus claris del Boccaccio. Studi sul Boccaccio 24: 3-6.

Caputo, V. (2008). Una galleria di donne illustri: il De mulieribus claris da Giovanni Boccaccio a Giuseppe Betussi. Cahiers d'Études Italiennes 8: |3|-| 48.

Cerbo, A. (1974). II De mulieribus claris di Giovanni Boccaccio. Arcadia 7: 5I-75.

Filosa, E. (2004). "Petrarca, Boccaccio e le mulieres clarae: dalla Familiare 21:8 al De mulieribus claris", Annali d'Italianistica 22: 381-393.

(2006). Intertestualità tra Decameron e De mulieribus claris: La tragica storia di Tisbe e Piramo, Heliotropia - An Online Journal of Research to Boccaccio Scholars: Vol. 3: Iss. I, Article I, pp. I-9.

(2007). "Boccaccio tra storia e invenzione: dal De fide uxorum erga viros di Valerio Massimo al De mulieribus claris", Romance Quarterly 54.3, pp. 219230.

(20I2). Tre studi sul "De mulieribus claris". Milano: LED.

Franklin, M. (2006). Boccaccio's Heroines. Power and Virtue in Renaissance Society. Burlington: Ashgate.

Hortis, A. (1877). Le donne famose descritte da Giovanni Boccaccio. Trieste: Stabilimento art. Tip. G. Caprin.

Jordan, C. (1987). Boccaccio's Famous Women: Gender and Civic Virtue in the De mulieribus Claris. Ambiguous Realities: 25-47.

Kolsky, S.(1993). La costituzione di una nuova figura letteraria. Intorno al De mulieribus claris di Giovanni Boccaccio. Testo 25: 36-52.

. (2003). The Genealogy of Women: Studies in Boccaccio's "De mulieribus claris". New York: Peter Lang. 
(2005). The Ghost of Boccaccio: Rewritings on "Famous Women" in Renassaince Italy. Turnhout: Brepols.

Meale, M. C. (1992). "Legends of Good Women in the European Middle Ages", Archiv das Studium der Neueren Sprachen und Literaturen 229. I, pp. 55-70.

Müller, R. (1992a). Ein Frauenbuch des frühen Humanismus. Untersuchungen zu Boccaccios 'De mulieribus claris'. Stuttgart: Franz Steiner Verlag.

. (1992b). Die jungfräuliche Künstlerin: Ein humanistisches Frauenideal in Boccaccios De mulieribus claris. Antike und Abendland: 124-I 32.

Nuvoloni, L. (2003). “De mulieribus claris: un frammento”, Studi sul Boccaccio 3I: 2326.

Pline l'Ancien (1985). Histoire naturelle. Livre XXXV. Parigi: Les Belles Letres.

Ricci, P. G. (1959). Studi sulle opere latine e volgari del Boccaccio. Rinascimento, $X$, I: 3-32.

Rico, F. (2002). El sueño del humanismo. De Petrarca a Erasmo. Barcelona: Destino.

Sciacovelli, A. D. (2005). Dal De viris illustribus al De mulieribus Claris. Verbum 7: 263279.

Torretta, L. (1902a). II 'Liber de claris mulieribus' di Giovanni Boccaccio. Giornale storico della letteratura italiana, 39: 252-292.

(1902b). II 'Liber de claris mulieribus' di Giovanni Boccaccio. Giornale storico della letteratura italiana, 40: 35-65.

Zaccaria, V. (1963). Le fasi redazionali del "De mulieribus claris", Studi sul Boccaccio, I: 252-332.

(1965). Appunti sul latino del Boccaccio nel "De mulieribus claris", Studi sul Boccaccio, III, 229-246.

(1978). La fortuna del De mulieribus claris del Boccaccio nel secolo XV: Giovanni Sabbadino degli Arienti, lacopo Filippo Foresti e le loro biografie femminili (1490-97). II Boccaccio nelle culture e letterature nazionali: 519-545.

Zappacosta, G. (1973). Per il testo del De mulieribus claris. I. II Cod. Laur. Pluteo XC sup. 98 e il testo dell'opera. Studi sul Boccaccio 7: 239-245. 
